\title{
Separate-activation models with variable base times: Testability and checking of cross-channel dependency
}

\author{
ROLF ULRICH and MARKUS GIRAY \\ Psychologisches Institut der Universitat Tubingen, Tubingen, Federal Republic of Germany
}

\begin{abstract}
If a subject is required to respond to either of two different target signals, reaction times (RTs) are especially fast when both target signals are presented. Separate-activation models can account for this finding. This model class assumes that each target signal is detected in a different channel and that the detection time of each channel is a random variable. If two different target signals are presented, RT is simply the lesser of the two detection times. Cumulative distribution functions of RTs are commonly used to test channel independence or, if channel independence is rejected, to evaluate the detection-time correlation. The present paper shows that it is important to consider the variability of the base time for all processes after the response has been decided on but before it has actually been carried out. It is shown that this variability influences the determination of the detection-time correlation. In addition, it is shown that Miller's (1982) test of separate-activation models versus coactivation models can also be applied to models with variable base times.
\end{abstract}

In many tasks, human observers monitor two distinguishable sources for a signal requiring a quick response. For example, in a bimodal detection task, the observer must respond as soon as a signal is presented on either of two modalities, say, vision and audition. On signal trials, only one signal (e.g., a tone or a flash) is presented, whereas on redundant-signal trials, both signals are presented simultaneously. Performance is studied in such a task by measuring the time (RT) between stimulation onset and response. The common finding is that RT is shorter for redundant-signal trials. This phenomenon has been called the "redundant signal effect" (Kinchla, 1974).

Miller (1982) has recently distinguished two model classes to explain the redundant-signal effect: the separateactivation and the coactivation models. Separate-activation models assume that the two signals are processed simultaneously within different channels and that each channel produces a separate activation (cf. Meijers \& Eijkman, 1977; Raab, 1962). The response is initiated as soon as an activation level is exceeded in either channel. In contrast to separate-activation models, coactivation models assume that the signals on the different channels produce a combined activation, and that the response is initiated as soon as this combined activation exceeds a criterion level.

Miller (1982) proposed a general test for the class of separate-activation models. In short, he has shown that

We thank Hans Colonius, the Editor, Wilhelm Glaser, Dominic Massaro, two anonymous reviewers, and Dirk Vorberg for helpful comments on an earlier draft of this paper. Some results presented here were reported at the 25. Tagung experimentell arbeitender Psychologen, Hamburg, 1983. Requests for reprints should be sent to Rolf Ulrich, Psychologisches Institut, Universität Tübingen, Friedrichstrasse 21, 7400 Tübingen, Federal Republic of Germany. the inequality $G_{X}(t)+G_{Y}(t) \geq G_{X Y}(t)$-where $G(t)$ is the probability that a response has been made by time $t$, and $X, Y$, and $X Y$ refer to conditions with a single-target signal on channel $C_{X}$, a single-target signal on channel $C_{Y}$, and signals on both channels $C_{X}$ and $C_{Y}$-must be satisfied for all values of $t$ if separate-activation models hold. A violation of this inequality supports coactivation models and rejects separate-activation models.

If the data satisfy the above inequality, and hence are consistent with the prediction of the separate-activation model, it is worthwhile investigating what type of separate-activation model might be consistent with the results obtained. In particular, recent papers have been concerned with the question of whether the detection times of the two channels are independent (Miller, 1982; Meijers \& Eijkman, 1977), and, if independency is rejected, whether they are positively or negatively correlated (Grice, Canham, \& Boroughs, 1984; van der Heijden, Schreuder, Maris, \& Neerincx, 1984). The baseline for this test rests on the following equality:

$$
m(t)=G_{X}(t)+G_{Y}(t)-G_{X}(t) * G_{Y}(t),
$$

where $m(t)$ denotes the predicted cumulative distribution functions (CDF) for redundant trials if the detection times are independent. Hence, if $m(t)$ and the observed CDF $G_{X Y}(t)$ coincide, then it is concluded that the detection times are independent (Grice, Canham, \& Boroughs, 1984; Meijers \& Eijkman, 1977; Miller, 1982; van der Heiden et al., 1984). Grice, Canham, \& Boroughs (1984) have even suggested a method to estimate the correlation of the detection times. In essence, this method yields a negative correlation if $G_{X Y}(t)>m(t)$ and a positive correlation if $G_{X Y}(t)<m(t)$.

The present work extends the test of Miller (1982). We 
proceed from the additional assumption of a variable base time for all processes after the response has been decided on but before it has actually been carried out (e.g., motor response times). It will be demonstrated that the variability of the base time influences the outcome of tests using Equality 1.

In addition it is shown that Miller's inequality can also be applied to separate-activation models with variable base times. We also provide a lower bound for $G_{X Y}(t)$. If $G_{X Y}(t)$ falls short of this lower bound, then the whole class of separate-activation models has to be rejected.

\section{SEPARATE-ACTIVATION MODELS WITH VARIABLE BASE TIMES}

Figure 1 shows the basic structure of the generalized separate-activation model. The model assumes two channels, $C_{X}$ and $C_{Y}$, operating in parallel. Each channel is linked with one and only one source (e.g., visual or auditory modalities). Each channel detects the relevant signal occurring in the corresponding source. The two channels, $C_{X}$ and $C_{Y}$, run into a final one, called the common channel. The common channel summarizes all the stages that follow stimulus detection. As soon as processing of the common channel ends, the subject's response occurs.

Let $X(Y)$ denote the detection time of target stimulus $S_{X}\left(S_{Y}\right)$ in channel $C_{X}\left(C_{Y}\right) . X$ and $Y$ are assumed to be random variables which might be negatively or positively correlated, or be independent. The random variable $B$ denotes the processing time (base time) of the common channel. According to the assumptions outlined, the RT for single signal trials is given by $\mathrm{RT}_{X}=X+B$ if only signal $S_{X}$ is presented, and by $\mathrm{RT}_{Y}=Y+B$ if only $S_{X}$ is presented. $\mathrm{RT}_{X Y}$ denotes the reaction time when both signals are presented, and is given by $\mathrm{RT}_{X Y}=\min (X, Y)+B$. The minimum of $X$ and $Y$ is denoted by $\min (X, Y)$, which, stated in other terms, is the interim between onset of signals and initiation of the common channel. Note that $\min (X, Y)$ is again a random variable. Since the mean of $\min (X, Y)$ must be smaller than or equal to either mean of $X$ and $Y$, the separate-activation model predicts faster RTs, on average, for redundant signal trials than for each type of single-target trials.

Theorem 1: Let $G_{X Y}(t), G_{X}(t)$, and $G_{Y}(t)$ be the $(o b-$ servable) CDFs of $R_{X Y}, R_{X}$, and $R_{Y}$, respectively. If the separate-activation model outlined above is true, then the following inequality must hold for all values of $t$ irrespective of whether or not the detection times $X$ and $Y$ are correlated (positively or negatively):

$$
G_{X}(t)+G_{Y}(t) \geq G_{X Y}(t) \geq \max \left[G_{X}(t), G_{Y}(t)\right] .
$$

(The proof of Inequality 2 is contained in Appendix A. ${ }^{1}$ ) Inequality 2 defines an upper and a lower bound for $G_{X Y}(t)$, and $\max \left[G_{X}(t), G_{Y}(t)\right]$ is the maximum value of the two CDFs $G_{X}(t)$ and $G_{Y}(t)$ at time $t$. If the observed $G_{X Y}(t)$ is less than this maximum value, then all separateactivation models have to be rejected. This lower bound has already been utilized by Grice, Canham, and Gwynne (1984, pp. 568-569) in order to evaluate distraction effects in redundant target trials. The left side of Inequality 2 puts an upper bound for $G_{X Y}(t)$. If $G_{X Y}(t)$ is greater than the sum of $G_{X}(t)$ and $G_{Y}(t)$, then all separateactivation models can be ruled out. It should be stressed that this test must hold (1) whether or not $X$ and $Y$ are dependent, (2) whether or not the base-time variance is large, and (3) whether or not the detection times are correlated with the base-time $B$; for example, there is some evidence that detection and motor times are positively correlated in a simple RT task (Ulrich \& Stapf, 1984). The left side of Inequality 2 agrees with Miller's (1982) inequality. This makes it certain that Miller's inequality can also be applied to separate-activation models with variable base times.

What can be said about $G_{X Y}(t)$ if the two detection times $X$ and $Y$ are independent? The following corollary provides an answer.

Corollary: If the processing times $X, Y$, and $B$ are stochastically independent variables, then for all values $t$, the inequality

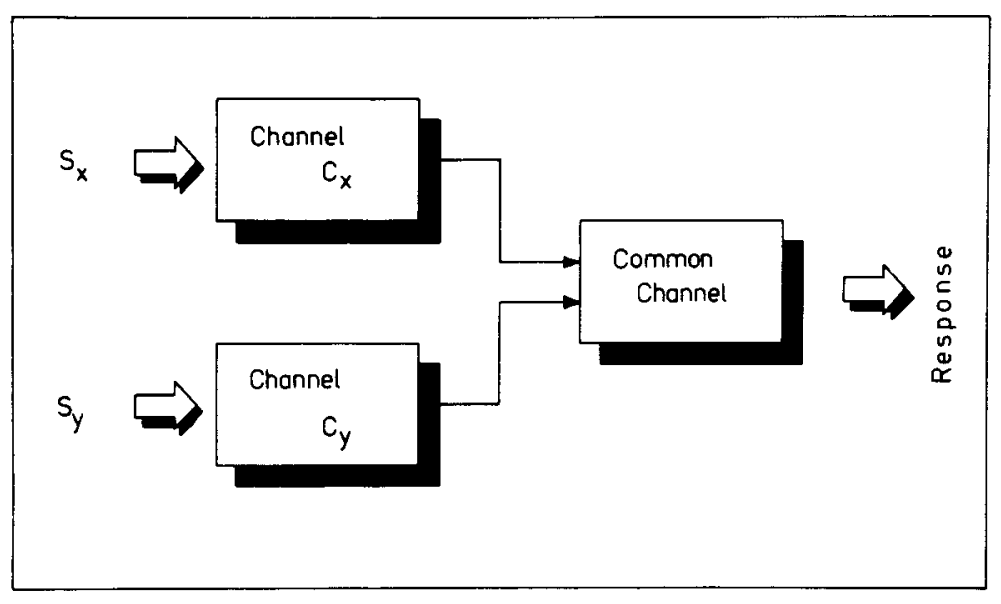

Figure 1. The separate-activation model with a final common stage. 


$$
G_{X}(t)+G_{Y}(t)-G_{X}(t) * G_{Y}(t) \geq G_{X Y}(t)
$$

must hold. (The proof is contained in Appendix B.)

Inequality 3 puts an upper limit on the observed $G_{X Y}(t)$ in the case of independent detection times. If the observed CDFs $G_{X}(t), G_{Y}(t)$, and $G_{X Y}(t)$ violate Inequality 3 , then all independent-channels-separate-activation models can be rejected.

Figure 2 summarizes the above testable predictions for fictitious data. This figure shows two hatched regions, $\mathrm{L}$ (lower region) and $\mathrm{U}$ (upper region). $\mathrm{U}$ is bounded above by $G_{X}(t)+G_{Y}(t)$ and bounded below by $G_{X}(t)+G_{Y}(t)-G_{X}(t) * G_{Y}(t)$, whereas $\mathrm{L}$ is bounded above by $G_{X}(t)+G_{Y}(t)-G_{X}(t) * G_{Y}(t)$ and bounded below by $\max \left[G_{X}(t), G_{Y}(t)\right]$. For all separate-activation models, the observed $G_{X Y}(t)$ must be placed anywhere within $U$ and/or L. If the processing times are assumed to be independent, then $G_{X Y}(t)$ must be placed anywhere within region $\mathrm{L}$.

\section{ILLUSTRATING THE TESTS BY MONTE-CARLO SIMULATIONS}

We conducted extensive simulations designed to demonstrate the effects of the base-time variance on the empirical determination of the detection-time correlation. In this simulation, it is assumed that $X, Y$, and $B$ are normally distributed random variables. Approximate normally distributed random numbers can be generated by using the method proposed by Box and Muller (1958), as shown by Equation 4:

$$
Z=\left[-2 * \log \left(U_{1}\right)\right]^{.5} * \cos \left(2 * \pi * U_{2}\right),
$$

where $Z$ is a normally distributed random deviate with zero mean and unit variance, and $U_{1}$ and $U_{2}$ are independent random variables between 0 and 1 from a rectangular distribution.
Let $Z_{1}$ and $Z_{2}$ be a pair of normal deviates generated by using Equation 4 . Then the two random numbers $D_{1}=Z_{1}$ and $D_{2}=$ corr $* Z_{1}+\left(1-\text { corr }^{2}\right)^{5} * Z_{2}$ represent a pair of deviates from a bivariate normal distribution with zero means, unit variances, and correlation coefficient corr (cf. Abramowitz \& Stegun, 1972, p. 953). Each deviate $D_{i}$ of the pair $\left(D_{1}, D_{2}\right)$ can be linearly transformed by using $T_{i}=S D_{i} * D_{i}+M_{i}$ to obtain a normal distributed random number $T_{i}$ with mean $M_{i}$ and standard deviation $S D_{i}, i=1,2$. The correlation coefficient between $T_{1}$ and $T_{2}$ equals corr, since any linear transformation of random variables does not change their correlation.

Ten thousand trials were used to simulate the CDF of $\mathrm{RT}_{X Y}$. In each trial, the following steps were performed: (1) A pair of correlated detection times $X$ and $Y$ were generated, as outlined above, with means $M_{X}$ and $M_{Y}$ and standard deviations $S D_{X}$ and $S D_{Y}$, respectively. (2) The smaller value of $X$ and $Y$ was determined; let $S$ denote this minimum. (3) A normal distributed base-time $B$ with mean $M_{B}$ and standard deviation $S D_{B}$ was generated. (4) $S$ and $B$ were added to produce $\mathrm{RT}_{X Y}$.

The CDFs of $\mathrm{RT}_{X}$ and $\mathrm{RT}_{Y}$ were obtained in a similar fashion. For example, to simulate $\mathrm{RT}_{X}$ one has to generate $X$ and $B$ in each trial and add these two random numbers. Ten thousand trials were used to simulate $G_{X}(t)$, and a further 10,000 trials were used for $G_{Y}(t)$.

Table 1 summarizes the results for several simulations with different model parameters. A small base-time variance was used for all simulations in Part 1 of Table 1 , whereas a large base-time variance was used for simulations in Part 2. The same means, $M_{X}, M_{Y}$, and $M_{B}$, were used for all simulations in Table 1. However, one set of standard deviations $\left(S D_{X}, S D_{Y}, S D_{B}\right)$ was used for Part 1 , and a different set was used for Part 2 . The standard deviations in each part were adjusted in such a way that, for ease of comparison and tabular presentation, the result-

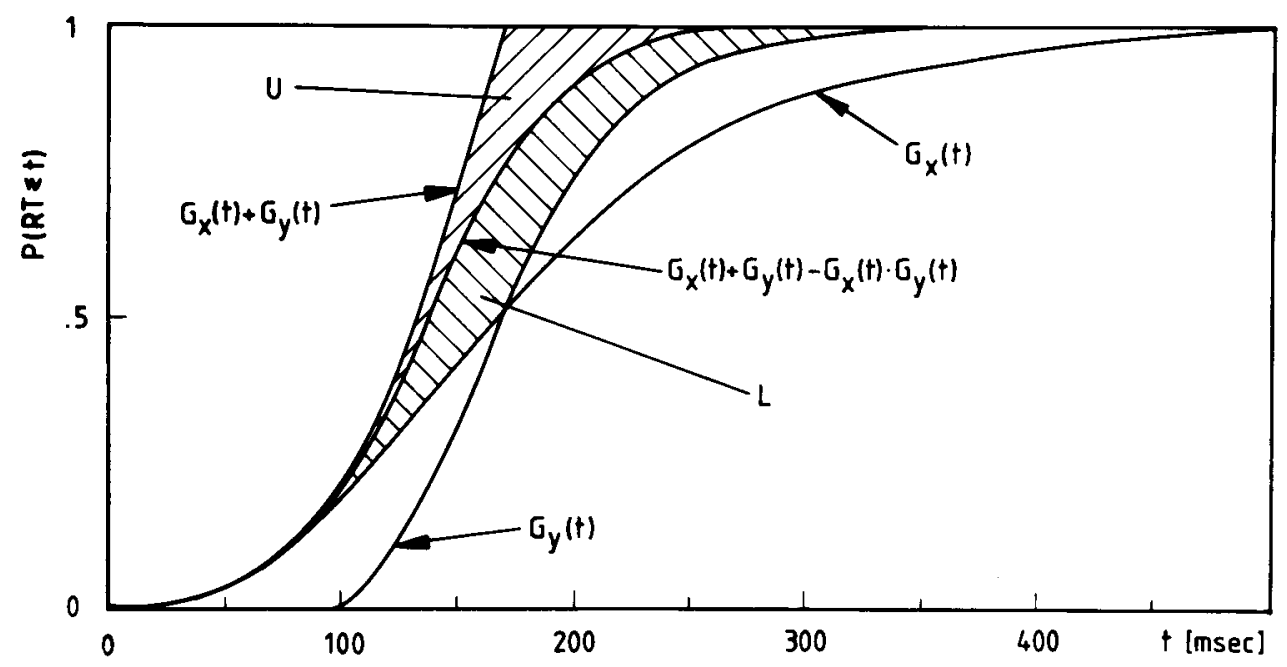

Figure 2. Illustration of the tests for the separate-activation model. Hatched regions ( $L$ and $U$ ) indicate permissible values of $G_{x \gamma}(t)$. These regions can be constructed using $G_{X}(t)$ and $G_{Y}(t)$. See text for a more detailed explanation. 
Table 1

Monte Carlo Studies of the Separate-Activation Model With Correlated Detection Times $X$ and $Y$

Selected Time Points $t$ (msec)

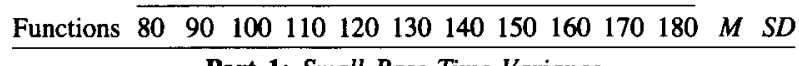
Part 1: Small Base-Time Variance

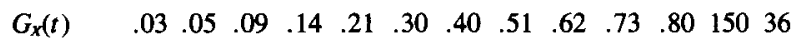

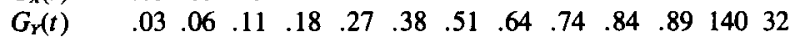

Bounds*

$\begin{array}{llllllllllll}u(t) & .05 & .11 & .20 & .33 & .47 & .68 & .91 & - & - & - & -\end{array}$

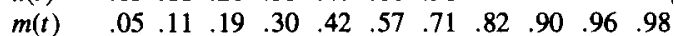

$\begin{array}{lllllllllllll}l(t) & & .03 & .06 & .11 & .18 & .27 & .38 & .51 & .64 & .74 & .84 & .89\end{array}$

$G_{X Y}(t)$

$\operatorname{corr}(X, Y)$

$\begin{array}{lllllllllllllll}-1.00 & .05 & .10 & .19 & .30 & .48 & .68 & .90 & 1.0 & 1.0 & 1.0 & 1.0 & 118 & 20\end{array}$

$\begin{array}{llllllllllllll}-0.75 & .06 & .10 & .19 & .30 & .47 & .67 & .82 & .94 & .99 & 1.0 & 1.0 & 120 & 22\end{array}$

$\begin{array}{llllllllllllll}-0.50 & .06 & .11 & .20 & .30 & .46 & .64 & .77 & .90 & .96 & .99 & 1.0 & 121 & 24\end{array}$

$\begin{array}{llllllllllllll}-0.25 & .05 & .11 & .20 & .30 & .45 & .61 & .74 & .86 & .94 & .97 & .99 & 123 & 26\end{array}$

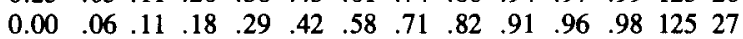

$\begin{array}{llllllllllllll}0.25 & .06 & .11 & .18 & .27 & .41 & .55 & .68 & .79 & .88 & .93 & .97 & 127 & 29\end{array}$

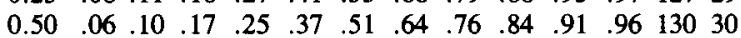

$\begin{array}{llllllllllllll}0.75 & .05 & .09 & .15 & .22 & .34 & .47 & .59 & .71 & .81 & .88 & .93 & 133 & 31\end{array}$

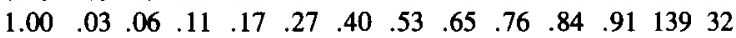

Part 2: Large Base-Time Variance

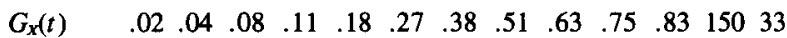

$\begin{array}{llllllllllllll}G_{Y}(t) & .03 & .06 & .12 & .19 & .28 & .39 & .51 & .63 & .74 & .82 & .89 & 140 & 32\end{array}$

Bounds*

$\begin{array}{llllllllllll}u(t) & .05 & .10 & .20 & .30 & .46 & .66 & .90 & - & - & - & -\dagger\end{array}$

$\begin{array}{llllllllllll}m(t) & .05 & .10 & .19 & .29 & .41 & .56 & .70 & .82 & .90 & .96 & .98\end{array}$

$\begin{array}{lllllllllllll}l(t) & & .03 & .06 & .12 & .19 & .28 & .39 & .51 & .63 & .74 & .82 & .89\end{array}$

$G_{x r}(t)$

$\operatorname{corr}(X, Y)$

$\begin{array}{llllllllllllll}-1.00 & .05 & .10 & .17 & .27 & .40 & .55 & .68 & .80 & .88 & .94 & .98 & 127 & 28\end{array}$

$\begin{array}{llllllllllllll}-0.75 & .05 & .09 & .16 & .26 & .39 & .54 & .66 & .79 & .87 & .93 & .97 & 128 & 28\end{array}$

$\begin{array}{llllllllllllll}-0.50 & .05 & .09 & .16 & .26 & .38 & .53 & .66 & .77 & .86 & .93 & .96 & 129 & 29\end{array}$

$\begin{array}{llllllllllllllll}-0.25 & .05 & .09 & .16 & .26 & .37 & .51 & .64 & .75 & .84 & .91 & .96 & 130 & 30\end{array}$

$\begin{array}{llllllllllllll}0.00 & .05 & .09 & .15 & .24 & .35 & .50 & .62 & .74 & .82 & .90 & .95 & 132 & 30\end{array}$

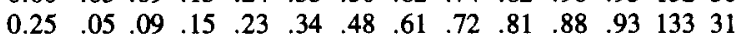

$\begin{array}{llllllllllllll}0.50 & .04 & .08 & .14 & .23 & .33 & .45 & .58 & .70 & .80 & .87 & .92 & 135 & 31\end{array}$

$\begin{array}{llllllllllllll}0.75 & .04 & .07 & .13 & .21 & .31 & .43 & .55 & .67 & .77 & .85 & .91 & 137 & 32\end{array}$

$\begin{array}{llllllllllllll}1.00 & .04 & .06 & .11 & .18 & .27 & .40 & .52 & .64 & .74 & .83 & .90 & 140 & 32\end{array}$

Note-Parameters used in Part 1: $M_{X}=90 \mathrm{msec}, M_{Y}=80 \mathrm{msec}, M_{B}$ $=60 \mathrm{msec}, S D_{x}=36 \mathrm{msec}, S D_{Y}=31 \mathrm{msec}, S D_{B}=2 \mathrm{msec}$. Parameters used in Part 2: $M_{X}=90 \mathrm{msec}, M_{Y}=80 \mathrm{msec}, M_{B}=$ $60 \mathrm{msec}, S D_{X}=25 \mathrm{msec}, S D_{Y}=20 \mathrm{msec}, S D_{z}=20 \mathrm{msec}$. *The bounds are $u(t)=G_{X}(t)+G_{Y}(t), m(t)=G_{X}(t)+G_{Y}(t)-G_{x}(t) * G_{Y}(t)$, and $l(t)=\max \left[G_{X}(t), G_{Y}(t)\right]$. †Values larger than 1.00 are omitted.

ing CDFs of both parts had about equal spread and location.

Consider the different aspects within each of the two parts. The first two rows show the CDFs for $\mathrm{RT}_{x}$ and $\mathrm{RT}_{Y}$. These CDFs were used to calculate the three different bounds for $G_{X Y}(t)$ according to Theorem 1 and the corrollary. These bounds appear beneath the CDFs of $\mathrm{RT}_{x}$ and $\mathrm{RT}_{Y}$. Then the subsequent rows show eight various CDFs of $\mathrm{RT}_{X Y}$, differing with regard to correlation $\operatorname{corr}(X, Y)$, beginning with $\operatorname{corr}(X, Y)=-1$, and increasing by 0.25 until $\operatorname{corr}(X, Y)=1$. Finally, it should be mentioned that the last two columns contain means and standard deviations of the corresponding RTs. After describing the various entries of Table 1 , we will discuss the main results of the simulations.
The obtained pattern of simulated RTs agrees fairly well with the pattern of results in a real experimental condition. "Statistical facilitation" (Rabb, 1962) is also obtained for this generalized version of the separateactivation model: both means $\mathrm{RT}_{X}$ and $\mathrm{RT}_{Y}$ are always greater than the corresponding mean of $\mathrm{RT}_{X Y}$. One can observe less "statistical facilitation" in the case of large base-time variance.

Detection-time correlation had a profound effect on $G_{X Y}(t)$. In both parts of Table $1, G_{X Y}(t)$ decreased with increasing $\operatorname{corr}(X, Y)$. For example, we obtained $G_{X Y}(140)=0.90$ for $\operatorname{corr}(X, Y)=-1$ in Part 1 . As $\operatorname{corr}(X, Y)$ increases, $G_{X Y}(140)$ decreases to 0.53 for $\operatorname{corr}(X, Y)=1$. Increasing $\operatorname{corr}(X, Y)$ also increases the mean and the standard deviation of $\mathrm{RT}_{X Y}$.

Consider now the two bounds $u(t)$ and $l(t)$. According to Theorem 1, all $G_{X Y}(t)$ s must be bounded above by $u(t)$ and below by $l(t)$. As one can see, the resulting regions in Parts 1 and 2 are quite narrow, suggesting a powerful test of the separate-activation model. Table 1 shows that all $G_{X Y}(t)$ s fall within this specified region. This test does not depend on the magnitude of the base-time variance.

The variance of the base time poses problems for a recent suggestion and application of a method to estimate the detection-time correlation (Grice, Canham, \& Boroughs, 1984, p. 452; van der Heijden et al., 1984). Grice, Canham, \& Boroughs suggested constructing a fourfold table from which a phi coefficient or tetrachoric correlation might be computed using the CDFs of $\mathrm{RT}_{x}$, $\mathrm{RT}_{Y}$, and $\mathrm{RT}_{X Y}$. The proportions of responding and not responding to the two types of signals on single signal trials provide estimates for the marginals of the table. The proportion of not responding when both stimuli are present determines one cell of the table. Since the table has only one degree of freedom, the remaining three cells can be filled.

We will illustrate this method using the data of Table 1 . For example, consider the time point $t=150 \mathrm{msec}$ in Part 1. A value of 0.94 is obtained for $G_{X Y}(150)$ and $\operatorname{corr}(X, Y)=-0.75$. The corresponding values for $G_{X}(150)$ and $G_{Y}(150)$ are 0.51 and 0.64 , respectively. Now one should use $G_{X Y}(t)$ to compute the desired proportion $P\left(\mathrm{RT}_{X} \geq 150\right.$ and $\left.\mathrm{RT}_{Y} \geq 150\right)=1-G_{X Y}(150)=$ $1-0.94=0.04$ of not responding at time $t=150 \mathrm{msec}$ when both stimuli are presented. This proportion is sufficient to complete the table for estimating the detectiontime correlation. For the numerical example, one computes a phi coefficient of -0.49 and a tetrachoric correlation coefficient of -0.73 . As one can see, the direction and the magnitude of the correlation coefficients agree fairly well with the corresponding correlation coefficient used to generate the CDF.

Let us now repeat the whole procedure for Part 2. A value of 0.79 was obtained for $G_{X Y}(150)$ at $\operatorname{corr}(X, Y)=$ -0.75 . The corresponding values for $G_{X}(150)$ and $G_{Y}(150)$ are 0.51 and 0.63 , respectively. The same computation as before yields a tetrachoric correlation of 0.19 and a phi coefficient of 0.12 . This time the estimated coefficients agree neither in direction nor in magnitude 
with the true detection-time correlation of -0.75 . These computations show that the proposed method of Grice, Canham, and Boroughs (1984) for estimating the detection-time correlation works well if the base-time variance is very small; however, it leads to wrong estimates if the base-time variance is relatively large. The simulations with a high base-time variance reveal a systematic overestimation of the detection-time correlation coefficient actually used in the simulation. A mathematical explanation of this systematic bias is given in Appendix C.

This consideration may also help to clarify some difficulties concerning the explanation of recent results obtained in a simple letter-detection paradigm (van der Heijden et al., 1984). Subjects were required to perform a speeded response if a target letter was presented at either of two different locations. On single-target trials, only one letter was presented at either of two different locations, whereas in redundant target trials, two target letters were presented at both locations. Their results showed a clear redundant-signal effect, and the authors favored a separate-activation explanation after applying Miller's (1982) test. In a further analysis, the authors investigated the direction of the detection-time correlation with the method outlined above. The analysis revealed a "rather unexpected pattern" (van der Heijden et al., 1984, p. 582): The observed $G_{X r}(t)$ for redundant signal trials exceeds the base-line $m(t)$ for small values of $t$ but falls short of this base line for intermediate and larger values of $t$. Hence, negative correlation coefficients were estimated for small values of $t$ and positive ones were estimated for larger values of $t$. Given our analysis, their results are consistent with negatively correlated detection times for all values of $t$, since $G_{X Y}(t)$ may fall short of $m(t)$ if the detection times are negatively correlated, as Part 2 of Table 1 shows. That is, if $G_{X Y}(t)$ falls short of $m(t)$, one cannot reject a negative detection-time correlation. Clearly, this interpretation requires that the basetime variance be relatively large compared with the variance of the detection-times. However, this requirement is not well supported empirically (Ulrich \& Stapf, 1984; Wing \& Kristofferson, 1973).

\section{CONCLUSION}

The present paper considered separate-activation models with variable base times. It was shown that Miller's (1982) upper bound $G_{X Y}(t) \leq G_{X}(t)+G_{Y}(t)$ could also be applied to check this more general model class against coactivation models. In addition, we provided a lower bound which may be used, for example, to reveal inhibitory effects upon RT in redundant signal trials (cf. Ueno, 1977). The variability of the base time does not influence the outcome of these two tests.

However, this variability influences the outcome of more specific questions regarding the dependence of the detection times: (1) It was shown that the base line, $G_{X}(t)+G_{Y}(t)-G_{Y}(t) * G_{X}(t)$, usually used to check independence of the detection times, can be applied only if one proceeds from a zero base-time variability. (2) Estimates of the detection-time correlation are influenced by the magnitude of the base-time variability. In general, the detection-time correlation will be overestimated if one applies the method suggested by Grice, Canham, and Boroughs (1984). The degree of overestimation depends largely on the magnitude of the base-time variability. Accurate estimates result with a zero basetime variability.

\section{REFERENCES}

Abramowitz, M., \& Stegun, I. A. (1972). Handbook of mathematical functions with formulas, graphs, and mathematical tables. New York: Dover.

Box, G. E. P., \& Muller, M. E. A. (1958). A note on the generation of random normal deviates. Annals of Mathematical Statistics, 29, 610-613.

Grice, G. R., Canham, L., \& Boroughs, J. M. (1984). Combination rule for redundant information in reaction time tasks with divided attention. Perception \& Psychophysics, 35, 451-463.

Grice, G. R., Canham, L., \& GWrnne, J. W. (1984). Absence of a redundant-signals effect in a reaction time task with divided attention. Perception \& Psychophysics, 36, 565-570.

KINCHLA, R. (1974). Detecting target elements in multielement arrays: A confusability model. Perception \& Psychophysics, 15, 149-158. Mejuers, L. M. M., \& Eijkman, E. G. J. (1977). Distributions of simple RT with single and double stimuli. Perception \& Psychophysics, 22, 41-48.

Miller, J. (1982). Divided attention: Evidence for coactivation with redundant signals. Cognitive Psychology, 14, 247-279.

RAAB, D. H. (1962). Statistical facilitation of simple reaction time. Transaction of the New York Academy of Sciences, 24, 574-590.

TownsEnd, J. T., \& AsHBY, F. G. (1983). Stochastic modeling of elementary psychological processes. Cambridge: Cambridge University Press.

UENO, T. (1977). Reaction time as a measure of temporal summation at suprathreshold levels. Vision Research, 17, 227-232.

ULRICH, R., \& STAPF, K. H. (1984). A double response paradigm to study stimulus intensity effects upon the motor system. Perception \& Psychophysics, 36, 545-558.

van der Heijden, A. H. C., Schreuder, R., Maris, L., \& Neerincx, M. (1984). Some evidence for positively correlated separate activation in a simple letter-detection task. Perception \& Psychophysics, 36, 577-585.

Wing, A. M., \& Kristofferson, A. B. (1973). Response delays and the timing of discrete motor responses. Perception \& Psychophysics, 14, 5-12.

\section{NOTE}

1. The reader should note that the left-hand side of Inequality 2 is the identical inequality already proved by Miller (1982, p. 253). However, Miller (1982) did not explicitly state whether or not this inequality was also valid for separate-activation models with a variable base time. Hence, we tried to make certain that his inequality could also be applied to models with a variable base time.

\section{APPENDIX A}

Proof of the Left-Hand Side of Inequality 2

Since the equation $\min (X, Y)+B=\min (X+B, Y+B)$ must hold, we can write

$$
\begin{aligned}
\mathrm{RT}_{X Y} & =\min (X, Y)+B \\
& =\min (X+B, Y+B) \\
& =\min \left(\mathrm{RT}_{X}, \mathrm{RT}_{Y}\right),
\end{aligned}
$$


$G_{X Y}(t)$

$=P\left(\mathrm{RT}_{X} \leq t\right)+P\left(\mathrm{RT}_{Y} \leq t\right)-P\left(\mathrm{RT}_{X} \leq t\right.$ and $\left.\mathrm{RT}_{Y} \leq t\right)$.

Now we use Miller's (1982, p. 253) argumentation, $P\left(\mathrm{RT}_{x}\right.$ $\leq t$ and $\left.\mathrm{RT}_{Y} \leq t\right) \geq 0$, which yields the prediction

$$
\begin{aligned}
G_{X Y}(t) & \leq P\left(\mathrm{RT}_{X} \leq t\right)+P\left(\mathrm{RT}_{Y} \leq t\right) \\
& \leq G_{X}(t)+G_{Y}(t) .
\end{aligned}
$$

The proof of the left-hand side of Inequality 2 is complete.

\section{Proof of the Right-Hand Side of Inequality 2}

If $G_{X Y}(t) \geq G_{X}(t)$ and $G_{X Y}(t) \geq G_{Y}(t)$ is true, then $G_{X Y}(t) \geq$ $\max \left[G_{X}(t), G_{Y}(t)\right]$ must also be true. Hence, we have to prove each of the two inequalities $G_{X Y}(t) \geq G_{X}(t)$ and $G_{X Y}(t) \geq G_{Y}(t)$ separately. Take, for example, $G_{X Y}(t) \geq G_{X}(t)$ :

Above we showed

$$
G_{X Y}(t)=G_{X}(t)+G_{Y}(t)-P\left(\mathrm{RT}_{X} \leq t \text { and } \mathrm{RT}_{Y} \leq t\right) \text {, }
$$

which can be rewritten, using conditional probability $P(\mathrm{~A}$ and $B)=P(\mathrm{~A} \mid \mathrm{B}) P(B)$, as

$$
\begin{aligned}
G_{X Y}(t) & =G_{X}(t)+G_{Y}(t)-P\left(\mathrm{RT}_{X} \leq t \mid \mathrm{RT}_{Y} \leq t\right) G_{Y}(t) \\
& =G_{X}(t)+G_{Y}(t)\left[1-P\left(\mathrm{RT}_{X} \leq t \mid \mathrm{RT}_{Y} \leq t\right)\right] .
\end{aligned}
$$

Since $P\left(\mathrm{RT}_{x} \leq t \mid \mathrm{RT}_{r} \leq t\right)$ can maximally be equal to one, it follows that $G_{Y Y}(t) \geq G_{x}(t)$ must be true.

In an analogous manner, one can prove that $G_{X Y}(t) \geq G_{Y}(t)$ if one substitutes $P\left(\mathrm{RT}_{Y} \leq t \mid \mathrm{RT}_{X} \leq t\right) G_{X}(t)$ for $P\left(\mathrm{RT}_{X} \leq t\right.$ and $\left.\mathrm{RT}_{Y} \leq t\right)$ in Equation $\mathrm{A} 1$. The proof is complete.

\section{APPENDIX B}

To simplify matters, we write $S$ for $\min (X, Y)$. Thus, $\mathrm{RT}_{X Y}$ equals the sum $S+B$; then the CDF of $\mathrm{RT}_{X Y}$ is given by

$$
\begin{aligned}
G_{X Y}(t) & =P(S+B \leq t) \\
& =\iint_{x+y \leq t} f_{s}(x) f_{B}(y) d x d y \\
& =\int_{0}^{t} F_{S}(t-y) f_{B}(y) d y,
\end{aligned}
$$

where $F_{S}(t)$ and $f_{B}(t)$ are the CDF and the density function of $S$ and $B$, respectively. Since $S$ is the minimum of $X$ and $Y$,

$$
F_{s}(t)=P(X \leq t)+P(Y \leq t)-P(X \leq t \text { and } Y \leq t) .
$$

It is assumed that $X$ and $Y$ are stochastically independent variables; hence,

$$
F_{S}(t)=F_{X}(t)+F_{V}(t)-F_{X}(t) F_{Y}(t)
$$

where $F_{X}(t)$ and $F_{Y}(t)$ are the CDFs of $X$ and $Y$, respectively.

Inserting Equation B2 into Equation B1 yields

$$
\begin{aligned}
G_{X Y}(t)= & \int_{0}^{t}\left[F_{X}(t-y)+F_{Y}(t-y)-F_{X}(t-y) F_{Y}(t-y)\right] f_{B}(y) d y \\
= & \int_{0}^{t} F_{X}(t-y) f_{B}(y) d y+\int_{0}^{t} F_{Y}(t-y) f_{B}(y) d y \\
& -\int_{0}^{t} F_{X}(t-y) F_{Y}(t-y) f_{B}(y) d y
\end{aligned}
$$

The first two terms on the right side of the last expression are the convolution integrals (cf. Townsend \& Ashby, 1983, pp. 30) of the sums $X+B$ and $Y+B$, respectively. Therefore, we can rewrite the last expression

$$
G_{X Y}(t)=G_{X}(t)+G_{Y}(t)-\int_{0}^{t} F_{X}(t-y) F_{Y}(t-y) f_{B}(y) d y .
$$

The corollary states that the following inequality should hold if $X$ and $Y$ are independent:

$$
G_{X}(t)+G_{Y}(t)-G_{X}(t) G_{Y}(t) \geq G_{X Y}(t) .
$$

Inserting Equation $\mathrm{B} 3$ in the right-hand side, simplifying, and rewriting yields

$$
\begin{gathered}
\int_{0}^{t} F_{X}(t-y) F_{Y}(t-y) f_{B}(y) d y-G_{X}(t) G_{Y}(t) \geq 0 \\
\int_{0}^{t} F_{X}(t-y) F_{Y}(t-y) f_{B}(y) d y \\
-\left[\int_{0}^{t} F_{X}(t-y) f_{B}(y) d y\right] \cdot\left[\int_{0}^{t} F_{Y}(t-y) f_{B}(y) d y\right] \geq 0 .
\end{gathered}
$$

This inequality is easily shown to be true. To this end, we define two functions, $h_{x}(y)$ and $h_{r}(y)$ :

$$
h_{x}(y)=\left\{\begin{array}{l}
F_{x}(t-y) \text { for } y<t, \\
0 \text { otherwise }
\end{array} h_{Y}(y)=\left\{\begin{array}{l}
F_{\mathrm{Y}}(t-y) \text { for } y<t, \\
0 \text { otherwise. }
\end{array}\right.\right.
$$

We rewrite Equation B4, using the definitions $h_{x}(y)$ and $h_{Y}(y)$ :

$$
\begin{aligned}
& \int_{0}^{\infty} h_{X}(y) h_{Y}(y) f_{B}(y) d y \\
& \quad-\left[\int_{0}^{\infty} h_{X}(y) f_{B}(y) d y\right] \cdot\left[\int_{0}^{\infty} h_{Y}(y) f_{B}(y) d y\right] \geq 0 .
\end{aligned}
$$

Note that the integrals are expectations of the random variables $h_{X}(B) * h_{Y}(B), h_{X}(B)$, and $h_{Y}(B)$, respectively. (One should note that any function of $B$ must be again a random variable.) Taking this consideration into account yields

$$
\begin{gathered}
E\left[h_{X}(B) h_{Y}(B)\right]-E\left[h_{x}(B)\right] \cdot E\left[h_{Y}(B)\right] \geq 0 \\
\operatorname{cov}\left[h_{X}(B), h_{Y}(B)\right] \geq 0,
\end{gathered}
$$

where the last expression is the covariance of $h_{x}(B)$ and $h_{Y}(B)$. Since both $h_{x}(y)$ and $h_{Y}(y)$ decrease with $y$, it must always be true that the covariance of $h_{X}(B)$ and $h_{Y}(B)$ is equal to or greater than zero. The proof is complete.

\section{APPENDIX C}

One outstanding result of the simulations is that the estimates of the detection-time correlations show a positive bias, that is, the estimates are always larger than the corresponding correlation coefficients used in the simulations. This effect is especially salient in the case of high base-time variance. This may be a little surprising, since, intuitively, one might expect that an added random variable simply attenuates whatever detection-time correlation coefficient was used in the simulation. Since this effect turns out to be systematic, a mathematical explanation of it may be helpful to correct one's intuition.

For a simple explanation, the reader should first notice that the relation $\mathrm{RT}_{X Y}=\min (X, Y)+B=\min (X+B, Y+B)=$ 
$\min \left(\mathrm{RT}_{X}, \mathrm{RT}_{Y}\right)$ must hold, irrespective of whether or not $X$ and $Y$ are correlated and whether or not the variance of $B$ is large. This relation shows that $\mathrm{RT}_{X Y}$ can actually be conceived as the minimum of $\mathrm{RT}_{X}$ and $\mathrm{RT}_{Y}$. Next, since the estimation procedure of Grice, Canham, and Boroughs (1984) utilizes RTs, the obtained estimates evaluate the correlation of $\mathrm{RT}_{X}$ and $\mathrm{RT}_{Y}$ in redundant signal trials instead of the desired correlation of $X$ and $Y$. It is obvious, then, that $\operatorname{corr}\left(\mathrm{RT}_{X}, \mathrm{RT}_{Y}\right)>\operatorname{corr}(X, Y)$ if $\operatorname{var}(B)>0$, as the following mathematical analysis shows:

To keep the analysis simple, we assume equal variances of $X$ and $Y$, that is, $\operatorname{var}(X)=\operatorname{var}(Y)$. Now the correlation of $\mathrm{RT}_{X}$ and $\mathrm{RT}_{Y}$ is given by

$$
\operatorname{corr}\left(\mathrm{RT}_{X}, \mathrm{RT}_{Y}\right)=\frac{\operatorname{cov}\left(\mathrm{RT}_{X}, \mathrm{RT}_{Y}\right)}{S D\left(\mathrm{RT}_{X}\right) S D\left(\mathrm{RT}_{Y}\right)} .
$$

Since $\mathrm{RT}_{X}=X+B, \mathrm{RT}_{Y}=Y+B$, and $\operatorname{var}(X)=\operatorname{var}(Y)$, we have

$$
\operatorname{corr}\left(\mathrm{RT}_{X}, \mathrm{RT}_{Y}\right)=\frac{\operatorname{cov}(X+B, Y+B)}{\operatorname{var}(X+B)} .
$$

The numerator of the last expression can be rewritten by using the distributive property of covariances (see Ulrich \& Stapf, 1984, p. 557) as $\operatorname{cov}(X+B, Y+B)=\operatorname{cov}(X, Y)+\operatorname{cov}(X, B)+$ $\operatorname{cov}(B, Y)+\operatorname{var}(B)$. Since the base time, $B$, was uncorrelated with either detection time in the above simulations, we have $\operatorname{cov}(X+B, Y+B)=\operatorname{cov}(X, Y)+\operatorname{var}(B)$ and $\operatorname{var}(X+B)=\operatorname{var}(X)$ $+\operatorname{var}(B)$. Substituting these results into the last expression yields:

$$
\operatorname{corr}\left(\mathrm{RT}_{X}, \mathrm{RT}_{Y}\right)=\frac{\operatorname{cov}(X, Y)+\operatorname{var}(B)}{\operatorname{var}(X)+\operatorname{var}(B)} .
$$

Since $\operatorname{var}(X)>0$, we can divide the numerator and the denominator of the above fraction by $\operatorname{var}(X)$, yielding

$$
\operatorname{corr}\left(\mathrm{RT}_{X}, \mathrm{RT}_{Y}\right)=\frac{\operatorname{corr}(X, Y)+\operatorname{var}(B) / \operatorname{var}(X)}{1+\operatorname{var}(B) / \operatorname{var}(X)}
$$

It can be easily shown, using Equation $\mathrm{C} 1$, that $\operatorname{corr}\left(\mathrm{RT}_{\boldsymbol{X}}, \mathrm{RT}_{Y}\right)$ increases with $\operatorname{var}(B)$ if all other things are kept equal, and hence no attenuation of $\operatorname{corr}(X, Y)$ will result if the base-time variance increases.

Next we will show that $\operatorname{corr}\left(\mathrm{RT}_{X}, \mathrm{RT}_{Y}\right)>\operatorname{corr}(X, Y)$ if $\operatorname{var}(B)>0$ :

$$
\operatorname{corr}\left(\mathrm{RT}_{X}, \mathrm{RT}_{Y}\right)>\operatorname{corr}(X, Y) .
$$

Inserting Equation $\mathrm{C} 1$ for $\operatorname{corr}\left(\mathrm{RT}_{X}, \mathrm{RT}_{Y}\right)$ yields

$$
\frac{\operatorname{corr}(X, Y)+\operatorname{var}(B) / \operatorname{var}(X)}{1+\operatorname{var}(B) / \operatorname{var}(X)}>\operatorname{corr}(X, Y) \text {. }
$$

After rearrangement and cancellation, we arrive at $\operatorname{corr}(X, Y)<1$, showing that the assumed direction of the inequality sign must hold.

In sum, the mathematical analysis shows that $(1) \operatorname{corr}(X, Y)$ is overestimated if one utilizes RTs for its estimation and (2) that the degree of this bias depends on the relative magnitude of the base-time variance (cf. Equation $\mathrm{C1}$ ).

(Manuscript received June 18, 1985; revision accepted for publication April 14, 1986.) 